\title{
A Novel FAND Nomogram to Predict the Risk of Hospital-Acquired Pneumonia after Acute Ischemic Stroke with Mechanical Thrombectomy
}

\section{Belynda Owoya Ochete}

Okayama Daigaku Yakugakubu Daigakuin Ishiyakugaku Sogo Kenkyuka Yakugakukei

\section{Linda Nyame}

China Pharmaceutical University

\section{Xiang Li}

China Pharmaceutical University

Yang Zou

Melbourne University

XueMei Li

The First Affiliated Hospital of Hunan Province; Peoples Hospital of Hunan

\section{Chaoping Huang}

Changsha Central Hospital

\section{Yajie Shan}

The First Affliated Hospital(People's Hospital of Hunan) Hunan Normal Univesity

\section{Ming Li}

China Pharmaceutical University

\section{Mako Ibrahim}

China Pharmaceutical Unviversity

\section{Qiong Jie}

Nanjing Medical University

\section{Yunxin Liu}

Nanjing Medical University

\section{Chun Ge}

Nanjing Medical University

Jue Hu

Changsha Central Hospital

\section{ZhiHong Zhao}

The First Affiliated Hospital(Peoples Hospital of HUnan) Hunan Normal University

Jianjun Zou ( $\square$ zoujianjun100@126.com )

Department of Clinical Pharmacology,Nanjing First Hospital,Nanjing Medical University https://orcid.org/0000-0003-0886-3153 
Research article

Keywords: Nomogram, Hospital-Acquired Pneumonia, Mechanical Thrombectomy, Acute Ischemic Stroke, Chinese

Posted Date: December 16th, 2019

DOI: https://doi.org/10.21203/rs.2.18959/v1

License: (c) (i) This work is licensed under a Creative Commons Attribution 4.0 International License.

Read Full License 


\section{Abstract}

Background: The timely prediction in the risk of Hospital-Acquired Pneumonia『HAP『in Acute Ischemic stroke (AIS) patients after Mechanical thrombectomy (MT) treatment is of high priority, given the rise in AIS mortality as a result. Although prior extensive research has been conducted in HAP preventive management and therapeutics, ischemic stroke patients are still at serious risk of contracting In-hospital pneumonia infections following certain medical procedures like Mechanical thrombectomy, a care standard for AIS patients. The predictive accuracy of patients with higher infection risk and adjusting therapeutic strategies accordingly will not only provide an enhanced preventive measure perspective but also significantly improve patient outcomes. Hence, our study was aimed at the validation and development of a novel predictive tool for risk stratification and individualized predictions of HAP occurrence in AIS patients after MT therapy.

Method: A multicenter retrospective study was executed with 405 AIS patients after undergoing MT treatment and admitted to the three Chinese stroke units. The major measure of outcome was to estimate the risk of HAP after MT through the integration of the following four predictors FBG, Age, NHISS, and Diastolic blood pressure (FAND) into a nomogram. Assessed on the multivariate logistic model, a nomogram was constructed, using the area under the receiver-operating characteristic curve to evaluate the discriminative performance and the Hosmer-Lemeshow test for risk prediction model calibration.

Results: Age(OR:1039; 95\%Cl 1.017-1.062; $\mathrm{p}=0.001)$, NIHSS(National Institutes of Health Stroke Scale) score on admission(OR:1.066; 95\% Cl: 1.030-1.103); $p<0.0001$ ), diastolic blood pressure(OR 1.023; $95 \% \mathrm{Cl}$ 1.006-1.040: $p=0.008)$, Fasting blood glucose(OR 1.1444; 95\% Cl 1.029-1.271; $p=0.013$ ) remained independent predictors of HAP integrated into the FAND nomogram after AIS Chinese patients received MT treatment. The Hosmer-Lemeshow goodness-of fit-test expressed good calibration(p-value: 0.496) and Area under the curve of 0.737 was exhibited for functional impairment prediction.

Conclusion: The FAND nomogram is a novel prognostic model developed and validated in Chinese AIS patients after MT treatment may aid in preventive measure strategies and predict poor patient outcomes.

\section{Background}

Acute ischemic stroke (AIS) is still a major cause of short and long term mortality and morbidity $[1,2]$. Mechanical thrombectomy (MT) as a medical procedure involving blood clot removal from blood vessels in the cerebral arteries has proven to be highly effective in AIS treatment[3]. However, post-therapeutic complications after MT for AIS treatment are frequent as a considerable amount of AIS-related deaths are directly attributed to suffered complications[4]. Hospital-acquired pneumonia (HAP) is defined as a lower respiratory tract infection in the lungs occurring roughly 48-72 hours after clinical admission and is rapidly emerging as a crucial patient safety concern[5]. HAP is the most dominant, dangerous and morbid AIS complication with an estimated mortality rate of about $30 \%$ and an 8 to $12 \%$ attributable mortality rate in stroke survivors; thereby increasing hospital admission time by about six days[6-9]. HAP 
incidence is, however, relative to the study population, considering the demographic increase in the elderly and longer life expectancy a further rise in the future number of patients experiencing complications after AIS is predictable[10]. The vast majority of comprehensive research conducted on HAP has primarily centered around diagnosis rather than eliminating infection prognosis which is equally important[11]. Although preventive intervention measures carried out by clinics show an impressive decrease in hospitalization and mortality rates, clinical complications have not been eliminated. HAP infections still see an expected increase in the next few years. Hence, early prediction of HAP onset after AIS is of great significance in providing a reasonable approach to clinical and therapeutic management[12-14].

Gaining insight into important factors in the prognosis of this condition might be challenging but highly necessary in accurately predicting patient outcomes, suggesting reasonable clinical and treatment management approach, and giving patients and their loved ones a better understanding of AIS $[3,15]$. Regardless of several scores constructed with the aim of predicting pneumonia emergence in stroke patients such as ISAN score, the PANTHERIS score, A2DS2(Age, Atrial fibrillation [AF], Dysphagia, Sex, Stroke Severity using National Institutes of Health Stroke Scale [NIHSS] score), Chumbler's score, Functional Bedside Aspiration Screen (FBAS score) and Stroke-Associated Pneumonia Score(acute ischemic stroke-associated pneumonia score, AIS-APS) there is however still a restriction on the acculturative effect in clinical care practice by its moderate predictive performance[16-21].

A nomogram is a reliable statistical tool that generates individualized approximation, faster prognostic prediction and continuous probability estimation of certain outcomes in a given patient, which can be developed from the mathematical visualization of complex formula through Cox proportional hazards analysis or multivariate logistic regression and by the incorporation of some continuous variables as a scoring system[22-24]. Nomograms are an integral constituent of modern clinical intervention and have been extensively integrated and validated in a wide array of medical applications[25-29]. However, to date, no nomogram models had been found to predict the risk of HAP after AIS with MT in Chinese patients.

The purpose of this study was the advancing and validating a nomogram comprising variables promptly accessible at the patient time of admission for the individualized prediction of HAP after MT which could directly aid individual treatment for AIS patients and provide relevant therapeutic preventive measures for patients with a higher risk of HAP.

\section{Methods}

\section{Study design, participants, and procedures}

A retrospective study was conducted based on data sequentially recorded from 405 patients between the period of January 2014 to February 2019. The AIS patients had been admitted to three-stroke units The First Affiliated Hospital (People's Hospital of Hunan Province), Nanjing First Hospital and Changsha Central Hospital respectively. After MT treatment, every patient gave consent to the use of their 
information for research purposes and scientific data collected was approved for research by the three hospital ethics committees in correlation with internal protocol and Helsinki Declaration. All studies executed under the consent of the Local Institutional Review Board.

Patients admitted with comprehensive clinical, demographic and laboratory information were solely considered for this research. The study's exclusion criteria were no diagnosis of in-hospital Pneumonia, age unknown or age $<18$, absence of FBG, anterior circulation stroke and TOAST, no Coronary heart disease medical history, therapy onset interval over $24 \mathrm{~h}$, incomplete data, and an unknown National Institutes of Health Stroke Scale(NIHSS) on admission.

The following were recorded, sex, age, medical history such as diabetes mellitus, hypertension, coronary artery disease, hyperlipidemia, transient ischemic stroke, previous cerebral infarction, atrial fibrillation, previous cerebral hemorrhage e.t.c, diastolic blood pressure, NIHSS score on admission and FBG(fasting blood glucose). The diagnosis of HAP after AIS treatment with MT through antibiotic treatment stimulation following admission was the clinical outcome.

\section{Statistical analysis}

The median value and interquartile range were set as continuous variables while using the Mann-Whitney U-test for univariate comparison to explore the cohort differences. The expression of categorical variables was alternatively expressed as the division of events numbers by the total amount except unknown or missing cases. Proportional differences were assessed by the $X^{\wedge} 2$ test or Fisher's exact test. The SPSS version 22.0 (IBM Corporation, Armonk, NY, USA), Stata version 13.0 (StataCorp, College Station, TX, USA) statistical software and the statistical software package R version 3.5.2 (R Development Core Team, Auckland, New Zealand) was used in the statistical analysis.

The FAND nomogram model was then constructed to predict the probability of hospital-acquired pneumonia after MT therapy. In the nomogram generation, a multivariate logistic regression analysis was conducted in a stepwise order which included, age, Fasting Blood Glucose (FBG), NIHSS score on admission and Diastolic BP (blood pressure) as pre-established variables, all with univariate analysis probability value at $<0.10$.

The foremost model was then selected based on the Akaike information criterion. The Condition Index ( $<30$ considered as non-significant) and Variation Inflation Factor Analysis (VIF, $<2$ considered as nonsignificant) of variable co-linearity combinations were used in the analysis of multivariate logistic. In the multivariate model, calculation of the odds ratio and its $95 \%$ interval of confidence were carried out for significantly associated primary endpoint variables.

Model performance was assessed by the method of discrimination (which is utilizing the start score to unrelated or divides pneumonia patients from patients without pneumonia) or the calibration method (Inhospital pneumonia prediction distance relative to actual patient outcome). The predictive accuracy of 
the nomogram model was evaluated through the calculation of the area below the receiver operating characteristic curve (AUC-ROC). Visual assessment was used in the test cohort through a calibration plot to determine the similarities between actual outcomes and outcomes predicted where, probability predicted was plotted against recorded pneumonia. Using a $45^{\circ}$ line as a perfect calibration indication, the match between the value predicted and the actual patient's risk was assessed. Furthermore, internal validation of the model was obtained with the use of 2000 bootstrap samples. Every test was two-sided and if the value of probability was $<0.05$ was considered statistically significant.

\section{Results}

Data from a total number of 405 AIS patients admitted to the three Chinese stroke units and treated with MT was complied. Patients were excluded from the study research for no In-hospital Pneumonia diagnosis $(n=9,2.2 \%)$, unknown NIHSS score on admission $(n=1 ; 0.2 \%)$, lack of FBG $(n=45 ; 11.1 \%)$, no anterior circulation stroke $(n=6 ; 1.5 \%)$, lack of TOAST $(n=11 ; 2.7 \%)$, no history with Coronary artery disease $(n=24 ; 5.9 \%)$, and patients $<18$ years old were also excluded from this research. Hence, the total number of only 305 patients with a complete data record useful in the nomogram generation participated in the study (Median age 72 years; IQR 62-79.5 years). The proportion of patients with in-hospital Pneumonia was calculated as $64.9 \%(198 / 305)$.

All clinical, laboratory and demographic data generated from the study population were stated in Table 1. Values included: age (67 versus $75 ; p<0.0001)$, NHISS score on admission (12 versus $16 ; p<0.0001$ ), Diastolic blood pressure (81 versus $86 ; p=0.017)$ and FBG $(5.17$ versus $6.71 ; p<0.0001)$ were all found to be significant in HAP prediction after AIS treatment with MT.

During the process of FAND nomogram development for the prediction of HAP after AIS treatment with MT, four non-categorical significant predictors were entered into a logistics regression model in the multivariate analysis: age(OR:1039; $95 \% \mathrm{Cl} 1.017-1.062 ; \mathrm{p}=0.001)$, NIHSS score on admission(OR:1.066; 95\%Cl: 1.030-1.103); $p<0.0001$ ), diastolic blood pressure(OR 1.023; 95\% Cl 1.006-1.040: $p=0.008)$, Fasting blood glucose(OR 1.1444; $95 \% \mathrm{Cl} 1.029-1.271 ; p=0.013)$. There was no significant co linearity observable for any of the four risk factors imputed in the multivariate regression analysis. Logistic regression model results were $\log (\mathrm{p}[\mathrm{x}] / 1-\mathrm{p}[\mathrm{x}])=-5.846+(0.038 \times$ age $)+(0.064 \times$ NIHSS score $)+(0.134$ $\times$ fasting blood glucose $)+(0.023 \times$ diastolic blood pressure $)$; where $p(x)$ was the probability of risk of inhospital pneumonia after ischemic stroke treated with MT.

The nomogram generation was based on assigning a graphic preliminary score to each of the 4 independent predictors with a point range within 1-100 and then summed up to generate a total score. Finally, they were converted into an individual risk of HAP after MT treatment of AIS is expressed in percentage between the range of $0-100 \%$. Predictions suggested a higher total nomogram score associated with higher probability of HAP after MT stroke treatment and a lower score associated with lower probability of HAP diagnosis after MT AIS treatment. 
The model was internally validated through the employment of 2000 bootstrap samples with AUC-ROC value of 0.737 ( $95 \% \mathrm{Cl} ; 0.679-0.795)$ (Figure 2). The age values with AUC of 0.655 (95\% Cl: $0.592-0.718$; $\mathrm{p}<0.0001$ ). The scores of NIHSS on admission displaying an AUC of 0.670 (95\% Cl: $0.606-0.734 ; \mathrm{p}<$ $0.0001)$ and the FBG with an AUC of $0.652(95 \% \mathrm{Cl}: 0.586-0.718 ; p<0.0001)$ all displayed diagnostic accuracy in hospital-acquired pneumonia patient identification. The overall number of patients with a risk probability of $<20 \%$ was $6 / 305(2.0 \%)$, and only one of these are acquired pneumonia ( 0.99 sensitivity, 0.05 specificity, 0.66 positive predictive value and, 0.83 negative predictive value). All patients showing a risk probability $<40 \%$ were $37 / 305(12.1 \%), 12$ of whom (32.4\%) acquired pneumonia (0.94 sensitivity, 0.23 specificity, 0.69 positive predictive value and 0.68 negative predictive value). Lastly, the total number of high-risk probability patients (i.e., $>80 \%$ ) was $77 / 305$ (25.2\%), a vast predominance in patients $(69 / 77$; $89.6 \%$ ) with poor prognosis ( 0.35 sensitivity, 0.93 specificity, 0.90 positive predictive value and 0.43 negative predictive value). The FAND nomogram model bias-corrected calibration plot illustrates good agreement between FAND nomogram predictors and verified HAP after MT treated AIS predictors. Hosmer-Lemeshow test P-value: 0.496 showing a good nomogram calibration in the goodness-of-fit test. Further, the mean variance inflation factor (VIF) was 1.03 indicating no multi-colinearity between predictors and considered non-significant.

\section{Discussion}

Ischemic stroke continues to be a leading cause of death and disability. An astonishing 87 percent of all stroke cases are ischemic resulting in as many as 6.7 million deaths worldwide[30]. Irrespective of the exponential advancement of MT devices and extensive recognition of the procedure as an advanced surgical alternative in AIS therapy due to comparative simplicity and efficacy, patients are still at risk of acquiring postoperative in-hospital pneumonia. In-hospital pneumonia continues to pose a major threat clinically considering the significant increase in mortality through patient immobilization, fever and, organ failure as a result of shock. Over 50,000 deaths (i.e. 1.6 deaths in 10,000 people) were reportedly due to pneumonia in the year 2015 alone[11]. The early prediction of In-Hospital pneumonia onset in AIS patients following MT treatment ought to be a prominent perspective on accurate and systematic therapeutic and clinical management[31-34].

Previous nomogram models and prognostic scores have identified Age and NHISS score as independent unfavorable outcome predictors in stroke patients. However, the categorization or dichotomization of predictors has been a major limitation as risk grouping system into 2 or 4 in independent continuous variables has proven to be statistically inefficient and significantly decrease predictive accuracy. Another important downside of dichotomization is the lack of in-category information incorporation often resulting in information diminution.

For proper estimation of specific clinical outcomes based on distinctive inputs at clinical interactions, a nomogram (graphical statistical predictive model) was constructed[19]. The FAND nomogram development was based on pre and post-treatment pre-established independent variables and through the combinational assessment of these four putative predictors readily available at time of admission; 
Age (OR: 1039; 95\%Cl 1.017-1.062; $p=0.001)$, Fasting Blood Glucose (OR: 1.066; 95\%Cl: 1.030-1.103); $p<0.0001$ ), Diastolic blood pressure (OR 1.023; $95 \% \mathrm{Cl} 1.006-1.040: p=0.008)$ and NIHSS score (OR $1.1444 ; 95 \% \mathrm{Cl} 1.029-1.271 ; p=0.013$ ) with NIHSS baseline score as the most proficient In-Hospital pneumonia predictor although age, FBG and diastolic blood pressure remained significant continuous variables. The nomogram presented a more dependable prognostic tool for the individualized prediction of HAP with a 5-95 percentile range (figure 1) in MT treated AIS Chinese patients[35-36]. The discriminative performance of the model was good, efficient and proved relevant even following the adjustment of other clinical and demographic variables.

In our study, a $>80 \%$ risk limit relative to a 0.90 positive predictive value was derived from the nomogram, providing a more accurate HAP risk after AIS treatment with MT prognosis. The lower risk limit of $<20 \%$ was obtained from the nomogram with a more negative predictive value of 0.66 , permitting an accurate probability exclusion in HAP diagnosis after AIS treatment with MT. Our study results suggested that the score created using variables at time of admission was feasible and reliable. For instance, the FAND nomogram allocated a $>95 \%$ adverse consequence probability in an 80-year-old patient(76 points) stroke patient, with diastolic blood pressure score of 112(48 points), FBG at a level of 12.5 (50 points) and NIHSS score of 25(50 points) and a score total of 224. Alternatively, the nomogram assigns a $<10 \%$ probability to a 30 -year-old ( 22.5 points) with diastolic blood pressure of 60 (13 points), FBG at a level of 4 (15 points) and NIHSS score of 5 (9 points) with a total score of 59.5 through score conversion into individual probability continuum, the FAND nomogram provides a more precise reclassification of HAP diagnostic outcome.

During the course of our study, we found that elderly patients 80 years old and above were predominantly at a particularly higher risk of HAP infection proving age to be a contributive factor to long-term mortality and pneumonia diagnosis in AIS patients[36]. These predispositions may be conveniently elucidated through medical conditions such as obstructive airway diseases or certain cardiovascular diseases such as high blood pressure, elevated cholesterol and coronary artery disease and also comorbid compromises in the immune system of the elderly. We also noticed an association between a higher NHISS score and impaired neurological and consciousness levels[37]. Patients experiencing severe neurological damage levels and those with consciousness level alterations have notably had a higher predisposition to Inhospital pneumonia diagnosis with previous study references. Some other important factors associated with In-hospital Pneumonia after MT treatment in AIS patients include; the use of antibiotics and glucocorticoid, Charlson Comorbidity Index (CCl) score and admission in the Intensive care unit(ICU). The management and functional evaluation of these several factors associated with hospital-acquired pneumonia diagnosis especially in elderly patients are recommended and a prime comprehensive possible-complication assessment ought to be carried out earlier in patient admission[38-42].

The FAND nomogram provided a functional decrease in the influence of alternative treatment prognosis since it was developed in compliance with data assembled from AIS patients treated with MT. Therefore, the nomogram could hold an advantage over previous models and prognostic scores comprehending the use of obsolete categorization in patient risk grouping for various risk predictor identification in prior 
models. Hence, providing better circumstantial information in the facilitation of timely detection in patients with a higher probability of acquiring In-hospital pneumonia aiding the relay of prognostic information to patients and their loved ones. The FAND nomogram acts as a visual tool beneficial in leading clinicians and patients to a better AIS treatment approach through individual stroke characterization and prognostications custom made to fit possible adverse effects.

Some research limitations were the comparatively small sample quantity and the retrospective nature of our study. Secondly, an important HAP predictor known as dysphasia was not included in the cohort and could influence the predictive accuracy of the model given dysphagia, age and NHISS score on admission is related to In-hospital pneumonia infection. Neuro-imaging predictors were also absent in this study, the presence of which could have provided higher discriminative performance and enhanced the nomogram's predictive accuracy in MT treated stroke patients. Also, during categorical grouping and predictive model generation, limited information on patient's ethnic, racial or geographical information was provided differences that could influence the HAP predictions. Further, our research data was collected based on administrative data manually compiled by a clinician; it may or may not have been neglected by clinical predictions. Lastly, external validation in different patient's cohort is required. Irrespective of the expressed limitations, our study is the first in our knowledge to develop and validate a prognostic nomogram for the prediction of HAP in Chinese AIS patients treated with MT.

The novel nomogram presented in our research was successfully used as a reliable and efficient tool in the prediction of In-Hospital pneumonia in AIS patients after receiving MT treatment. The prognosis provided by the FAND nomogram was constructed in compliance with possible adverse effects modeled through individual stroke characterization. In summary, this study suggests that demographic, laboratory and clinical predictors (like age, FBG, NIHSS admission score, and diastolic blood pressure) may be preferable and more reliable predictors of HAP in MT treated Chinese AIS patients which could result in management strategy enhancement and better therapeutic approach by clinicians.

\section{Conclusions}

The FAND nomogram provided useful and relatively accurate information through the integration of independent and non-categorical predictors for HAP diagnosis probability in Chinese acute ischemic stroke patients who underwent mechanical thrombectomy treatment.

\section{Abbreviations}

HAP: Hospital-acquired pneumonia; AIS: Acute Ischemic Stroke; MT: Mechanical Thrombectomy; AUC: The area under the curve; BP: Blood pressure; CCI: Charlson Comorbidity Index; ICU: Intensive care unit; $\mathrm{Cl}$ : Confidence interval; FBG: Fasting blood glucose; FAND: Fasting blood glucose, Age, MHSS score on admission and Diastolic blood pressure OR: Odds ratios; RCT: Randomized controlled trial; ROC: Receiver operating characteristic; SD: Standard deviation; TG: Triglyceride; VIF: Variation Inflation Factors 


\section{Declarations}

\section{Acknowledgments}

We would like to thank all patients, researchers, and institutions for their collaboration and contribution to this study. We acknowledge the Nanjing first Hospital, relevant clinicians, and investigators for their participation.

\section{Author's contributions}

BOO, LN and $Y Z$ contributed equally to this work. JJZ and ML concepted, designed and supervised the study. XML, CPH, YJS, JH and ZHZ acquired the data. BOO, XL and JJZ analyzed and interpreted the data, provided statistical analysis, had full access to all of the data in the study, and are responsible for the integrity of the data and the accuracy of the data analysis. BOO, LN and YZ drafted the manuscript, $\mathrm{MI}, \mathrm{QJ}, \mathrm{YXL}$ and CG critically revised the manuscript for important intellectual content. All authors read and approved the final manuscript.

\section{Funding}

This study was supported by the National Natural Science Foundation of China grant 81673511 , Jiangsu key Research and Development Plan grant BE2017613, Jiangsu Six Talent Peaks Project grant WSN-151, and Nanjing Medical Science and Technique Development Foundation grant QRX17020 and ZKX15027.

\section{Availability of data and materials}

The data sets in this study are available from the corresponding author on reasonable request.

\section{Ethics approval and consent to participate}

The study protocols were approved by the Ethics Committees of Nanjing First Hospital in accord with the Helsinki declaration and internal protocol. All patients have given their written informed consent.

\section{Consent for publication}

Not applicable. 


\section{Competing interests}

The authors have declared that they have no conflicts of interest regarding the content of this article.

\section{Author Details}

${ }^{1}$ School of Basic Medicine and Clinical Pharmacy, China Pharmaceutical University, Nanjing, China;

${ }^{2}$ Department of Clinical Pharmacology, Nanjing First Hospital, Nanjing Medical University, Nanjing, China;

${ }^{3}$ Faculty of Science, Melbourne University, Australia; ${ }^{4}$ Department of Neurology, The First Affiliated Hospital (People's Hospital of Hunan Province), Hunan Normal University, Changsha, China; 5 Department of Neurology, Changsha Central Hospital, Changsha, China; ${ }^{6}$ College of life Science and Technology, China Pharmaceutical University, Nanjing, China.

\section{References}

1. Benjamin EJ, Virani SS, Callaway CW, et al. A Report From the American Heart Association. Heart Disease and Stroke Statistics. 2018;137:67-492.

2. Gianni Turcato, Gianfranco Cervellin, Manuel Cappellari, et al. Early function decline after ischemic stroke can be predicted by a nomogram based on age, use of thrombolysis, RDW and NIHSS score at admission. Journal of Thrombolysis. 2016;43(3):394-400.

3. Goyal M, Menon BK, van Zwam WH, et al. HERMES collaborators. Endovascular thrombectomy after large-vessel ischemic stroke: a meta-analysis of individual patient data from five randomized trials. Lancet. 2016;387:1723-1731.

4. Yuetian Yu, Cheng Zhu, Chunyan Liu, et al. Effect of Prior Atorvastatin Treatment on the Frequency of Hospital Acquired Pneumonia and Evolution of Biomarkers in Patients with Acute Ischemic Stroke: A Multicenter Prospective Study. Hindawi. 2017(20):1-8.

5. Dian Baker PhD, RN a, Barbara Quinn MS, et al. Hospital Acquired Pneumonia Prevention Initiative-2: Incidence of non ventilator hospital-acquired pneumonia in the United States, Elsevier 2018;46(1):2-7.

6. Kumar S, Selim MH, Caplan LR. Medical complications after stroke. Lancet Neurol. 2010; 9:(1)105-118.

7. Katzan IL, Cebul RD, Husak SH, et al. The effect of pneumonia on mortality among patients hospitalized for acute stroke. Neurology. 2003;60(4):620-625.

8. Kumar, A. D., Dorsey, et al. Infections Present on Admission Compared with Hospital-Acquired Infections in Acute Ischemic Stroke Patients. Journal of Stroke and Cerebrovascular Diseases. 2013; 
22(8),582-589.

9. Klompas, M. Prevention of Intensive Care Unit-Acquired Pneumonia. Seminars in Respiratory and Critical Care Medicine. 2019;40(04):548-557.

10. Weimar C, Konig IR, Kraywinkel K, et al. Age and National Institutes of Health Stroke Scale Score within 6 hours after onset are accurate predictors of outcome after cerebral ischemia: development and external validation of prognostic models. Stroke. 2004;35:158-162.

11. Christian S. Marchello, Mark H. Ebell, Ariella P. Dale, et al. The Journal of the American Board of Family Medicine. 2019;32(2):234-247.

12. Ingeman A, Andersen G, Hundborg HH, et al. In-hospital medical complications, length of stay, and mortality among stroke unit patients. Stroke. 2011;42(11):3214-3218.

13. Katzan IL, Dawson NV, Thomas CL, et al. The cost of pneumonia after acute stroke. Neurology. 2007;68(22):1938-1943.

14. Koennecke HC, Belz W, Berfelde D, et al. Factors influencing in-hospital mortality and morbidity in patients treated on a stroke unit. Neurology. 2011;77(10):965-972.

15. Adams HP Jr, Davis PH, Leira EC, et al. Baseline NIH Stroke Scale score strongly predicts outcome after stroke: A report of the Trial of Org 10172 in Acute Stroke Treatment (TOAST). Neurology 1999;53:126-131

16. Harms H, Grittner U, Droge H, Meisel A et al. Predicting post-stroke pneumonia: the PANTHERIS score. Acta neurologica Scandinavica 2013;128(3):178-184.

17. Ji R, Shen H, Pan Y, et al. Novel risk score to predict pneumonia after acute ischemic stroke. Stroke. 2013;44(5):1303-1309.

18. Chumbler NR, Williams LS, Wells CK, et al. Derivation and validation of a clinical system for predicting pneumonia in acute stroke. Neuroepidemiology. 2010;34(4):193-199.

19. Smith CJ, Bray BD, Hoffman A, et al. Can a novel clinical risk score improve pneumonia prediction in acute stroke care? A UK multicenter cohort study. J Am Heart Assoc. 2015;4(1):001307.

20. Hoffmann S, Malzahn U, Harms H, et al. Development of a clinical score (A2DS2) to predict pneumonia in acute ischemic stroke. Stroke. 2012;43(10):2617-2623.

21. Virvidaki IE, Giannopoulos S, Nasios G, et al. Predictive value of a novel pragmatic tool for post-stroke aspiration risk: The Functional Bedside Aspiration Screen. Neurogastroenterology and motility : the official journal of the European Gastrointestinal Motility. 2019;31(10):13683. 
22. BaiLi Song, Xiang Liang Chen, Dan Tang, et al. External Validation of START nomogram to predict 3Month unfavorable outcome in Chinese acute stroke patients. The journal of stroke and cardiovascular diseases. 2019;28(6):1618-1622.

23. Cappellari, M, Mangiafico, S, Saia, V et al. ER-SICH Nomogram to Predict Symptomatic Intracerebral Hemorrhage After Thrombectomy for Stroke. Stroke. 2019;50(4):909-916

24. Manuel Cappellari, Gianni Turcato, Stefano Forlivesi, et al. STARTING-SICH Nomogram to Predict Symptomatic Intracerebral Hemorrhage After Intravenous Thrombolysis for Stroke. Stroke. 2018;49:0000 .

25. Manuel Cappellari, Gianni Turcato, Stefano Forlivesi, et al. The START nomogram for individualized prediction of the probability of unfavorable outcome after intravenous thrombolysis for stroke. Journal of Stroke. 2018;0(0):1-7.

26. Moulla Y, Lyros O, Adolf D, et al. A Nomogram Based on Clinical Factors to Predict the Serum Myoglobin Levels Following Bariatric Surgery. Obesity Surgery 2018;28:1697-1703.

27. Battersby NJ, Bouliotis G, Emmertsen KJ, et al. Development and external validation of a nomogram and online tool to predict bowel dysfunction following restorative rectal cancer resection: the POLARS score. Gut 2018;67:688-696.

28. Song W, Zhu ZG, Wu Q, et al. A nomogram to predict overall survival for biliary tract cancer. Cancer Management Research 2018;10:1535-1541.

29. Zhou X, Sun Z, Zhuang Y, et al. Development and Validation of Nomogram to Predict Acute Kidney Injury in Patients with Acute Myocardial Infarction Treated Invasively. International Journal of Science Reports 2018;8:9769.

30. Manuel Cappellari, Gianni Turcato, Stefano Forlivesi, et al. The START nomogram for individualized prediction of the probability of unfavorable outcome after intravenous thrombolysis for stroke. Journal of Stroke. 2018;0(0):1-7.

31. Ji R, Shen H, Pan Y, et al. Novel risk score to predict pneumonia after acute ischemic stroke. Stroke. 2013;44(5):1303-1309.

32. Chumbler NR, Williams LS, Wells CK, et al. Derivation and validation of a clinical system for predicting pneumonia in acute stroke. Neuroepidemiology. 2010;34(4):193-199.

33. Baker, D, Quinn, B et al. Hospital Acquired Pneumonia Prevention Initiative-2: Incidence of nonventilator hospital-acquired pneumonia in the United States. American Journal of Infection Control. 2018;46(1):27. 
34. Petroianni A, Ceccarelli D, Conti V, et al. Aspiration pneumonia. Pathophysiological aspects, prevention and management. Panminerva Med. 2006;48(4):231-239.

35. Perry L, Love CP. Screening for dysphagia and aspiration in acute stroke: a systematic review. Dysphagia. 2001;16(1):7-18.

36. Ji R, Shen H, Pan Y, et al. Novel risk score to predict pneumonia after acute ischemic stroke. Stroke. 2013;44(5):1303-1309.

37. Sellars C, Bowie L, Bagg J, et al. Risk factors for chest infection in acute stroke: a prospective cohort study. Stroke. 2007;38(8):2284-2291.

38. Aslanyan S, Weir CJ, Diener HC, et al. Pneumonia and urinary tract infection after acute ischemic stroke: a tertiary analysis of the GAIN International trial. The European Journal of Neurology. 2004;11(1):49-53.

39. Vargas M, Horcajada JP, Obach V, et al. Clinical consequences of infection in patients with acute stroke: is it prime time for further antibiotic trials. Stroke. 2006;37(2):461-465.

40. Ji R, Shen H, Pan Y, et al. Risk score to predict hospital-acquired pneumonia after spontaneous intracerebral hemorrhage. Stroke. 2014;45(9):2620-2628.

41. Walter U, Knoblich R, Steinhagen V, et al. Predictors of pneumonia in acute stroke patients admitted to a neurological intensive care unit. J Neurol. 2007;254(10):1323-1329.

42. Jing Jiao, Xiang-yun Yang, Zhen Li, et al. Incidence and Related Factors for Hospital-Acquired Pneumonia Among Older Bedridden Patients in China: A Hospital-Based Multicenter Registry Data Based Study. Frontiers in Public Health. 2019;7:221.

\section{Tables}

TABLE 1. Clinical, demographic and laboratory data of study population. 


\begin{tabular}{|c|c|c|c|}
\hline & No Pneumonia & Pneumonia & $P$ \\
\hline Patients, n (\%) & 107 & 198 & \\
\hline Age (years), median (IQR) & $67(58-75)$ & $75(64-81)$ & $<0.0001 * \#$ \\
\hline Sex, $\mathrm{n}(\%)$ & & & 0.860 \\
\hline Male, n (\%) & $67(62.6)$ & 126(63.6] & \\
\hline Female, n (\%) & $40(37.4)$ & $72(36.4)$ & \\
\hline \multicolumn{4}{|l|}{ Medical history, n (\%) } \\
\hline Hypertension & $70(65.4)$ & $147(74.2)$ & $0.105 *$ \\
\hline Diabetes mellitus & $19(17.8)$ & $42(21.2)$ & 0.472 \\
\hline Hyperlipidemia & $4(3.7)$ & $8(4.0)$ & $1.000 *$ \\
\hline Coronary artery disease & $16(15.0)$ & $46(23.2)$ & $0.086^{*}$ \\
\hline Atrial fibrillation & $27(30.7)$ & $56(37.3)$ & $0.141 *$ \\
\hline Transient ischemic attack & $0(0)$ & $3(1.5)$ & 0.554 \\
\hline Previous cerebral infarction & $22(20.6)$ & $42(21.2)$ & 0.894 \\
\hline Previous cerebral hemorrhage & $5(4.7)$ & $3(1.5)$ & 0.204 \\
\hline Smoking, n (\%) & & & 0.265 \\
\hline Never smoker & $61(57.0)$ & 117(59.4) & \\
\hline Former smoker & $8(7.5)$ & $24(12.2)$ & \\
\hline Current smoker & $38(35.5)$ & $56(28.4)$ & \\
\hline \multicolumn{4}{|l|}{ Baseline data } \\
\hline $\begin{array}{l}\text { NIHSS score on admission, median } \\
\text { (IQR) }\end{array}$ & $12(7-17)$ & $16(12-21)$ & $<0.0001 * \#$ \\
\hline Systolic BP, mmHg, median (IQR) & $138(123-156)$ & $144(130-159)$ & $0.130 \#$ \\
\hline Diastolic BP, mmHg, median (IQR) & $81(72-93)$ & $86(77-99)$ & $0.017 * \#$ \\
\hline INR, median (IQR) & $1.00(0.95-1.06)$ & $0.99(0.94-1.08)$ & $0.834 \#$ \\
\hline Creatinine, umol/L, median (IQR) & $\begin{array}{l}64.00(57.00- \\
80.00)\end{array}$ & $\begin{array}{l}74.00(61.0- \\
92.0)\end{array}$ & $0.006 * \#$ \\
\hline FBG, mmol/L, median (IQR) & $5.71(4.89-7.16)$ & $6.71(5.73-8.23)$ & $<0.0001 * \#$ \\
\hline Platelet count, $10^{9} / \mathrm{L}$, median (IQR) & $179(141-224)$ & 176(143-225) & \\
\hline $\mathrm{TC}, \mathrm{mmol} / \mathrm{L}$, median (IQR) & $4.30(3.54-5.09)$ & $4.25(3.49-4.97)$ & $0.646 \#$ \\
\hline TG, mmol/L, median (IQR) & $1.08(0.77-1.52)$ & $1.04(0.74-1.50)$ & $0.517 \#$ \\
\hline LDL, mmol/l, median (IQR) & $2.69(2.01-3.29)$ & $2.51(1.96-3.09)$ & $0.217 \#$ \\
\hline HbA1c, \%, median (IQR) & $5.80(5.50-6.30)$ & $5.90(5.50-6.40)$ & $0.662 \#$ \\
\hline UA, umol/l, median (IQR) & $\begin{array}{l}267.00(227.00- \\
371.00)\end{array}$ & $\begin{array}{c}\text { 319.50(221.6- } \\
385.5)\end{array}$ & $0.165 \#$ \\
\hline anterior circulation stroke, n (\%) & $88(82.2)$ & 157(79.3) & 0.536 \\
\hline posterior circulation stroke, n (\%) & $21(19.6)$ & $44(22.2)$ & 0.597 \\
\hline TOAST classification & & & 0.119 \\
\hline Large artery atherosclerosis, n (\%) & $42(39.3)$ & $55(27.8)$ & \\
\hline Cardioembolism, n (\%) & $45(42.1)$ & 115(58.1) & \\
\hline Others, n (\%) & $20(18.6)$ & $11(14.1)$ & \\
\hline intravenous thrombolysis, n (\%) & & & $0.095 *$ \\
\hline No thrombolysis & $70(65.4)$ & $110(55.6)$ & \\
\hline Thrombolysis & $37(34.6)$ & $88(44.4)$ & \\
\hline $\begin{array}{l}\text { Interval from onset to treatment, min, } \\
\text { median (IQR) }\end{array}$ & $325(243-450)$ & $270(198-380)$ & $0.006 * \#$ \\
\hline
\end{tabular}

INR International normalized ratios, FBG Fasting blood glucose, TC total cholesterol, TG triglyceride, LDL Low density lipoprotein, HbAc1 Glycated hemoglobin, UA Uric Acid, TOAST Trial of ORG 10172 in Acute Stroke Treatment. *included into the multiple logistic regression models $(P<0.1)$. Additionally, traditional stroke risk factor such as Atrial fibrillation was added into the model. \# Calculated using Mann-Whitney U test. 
TABLE 2. Significant predictors of in-hospital pneumonia after acute ischemic stroke in Chinese patients treated with MT.

\begin{tabular}{lccccc}
\hline & OR & Error & Wald & P & 95\% CI \\
\hline Age & 1.039 & 0.011 & 3.45 & 0.001 & $1.017-1.062$ \\
NIHSS on admission & 1.066 & 0.018 & 3.68 & P<0.0001 & $1.030-1.103$ \\
Diastolic blood pressure & 1.023 & 0.009 & 2.64 & 0.008 & $1.006-1.040$ \\
FBG & 1.144 & 0.062 & 2.49 & 0.013 & $1.029-1.271$ \\
\hline $\begin{array}{l}\text { NIHSS, National Institutes of Health Stroke Scale; FBG, fasting blood glucose; BP, } \\
\text { blood pressure. }\end{array}$
\end{tabular}

\section{Figures}

\section{Figure 1}

The nomogram presented a more dependable prognostic tool for the individualized prediction of HAP with a 5-95 percentile range in MT treated AIS Chinese patients

\section{Figure 2}

The model was internally validated through the employment of 2000 bootstrap samples with AUC-ROC value of $0.737(95 \% \mathrm{Cl} ; 0.679-0.795)$

\section{Figure 3}

The FAND nomogram model bias-corrected calibration plot illustrates good agreement between FAND nomogram predictors and verified HAP after MT treated AIS predictors. 